Indian J. Phys. 83 (4) 473-477 (2009)

\title{
Nanoparticle size characterization by laser light scattering
}

\author{
A Gogoi*, G A Ahmed and A Choudhury \\ Optoelectronics and Photonics Laboratory, Department of Physics, \\ Tezpur University, Tezpur-784 028, Assam, India \\ E-mail : ankur@tezu.ernet.in
}

\begin{abstract}
Spherical semiconductor nanoparticles (ZnS) were specially fabricated by an inexpensive chemical route. The scattering profile of the nanoparticles was investigated by laser light scattering technique. A beam of polarized light from a diode laser $\left(\lambda_{0} \approx 630 \mathrm{~nm}\right)$ was allowed to fall on the nanospheres embedded in flexible host matrix Polyvinyl Alcohol (PVA). The light scattered from the samples were detected by means of analyzer mounted photodiode array from $10^{\circ}$ to $170^{\circ}$ in steps of $1^{\circ}$. Signals from the detectors were interfaced with a high resolution data acquisition system and the whole experiment was carried out in differential mode. Size of the nanoparticles was obtained by using Mie theory and verified by $T$-matrix approach. The results obtained agree with the XRD and TEM results.
\end{abstract}

Keywords : Nanoparticles, laser light scattering, photodiode, Mie theory.

PACS Nos. : 78.67.Bf, 85.60.Dw, 42.25.Fx, 42.68.Mj

\section{Introduction}

Light scattering experiments have emerged as one of the most promising and useful technique now a days for in situ measurement of size, size distribution and shape of low dimensional nanostructrues. The scattering angle dependence of all 16 elements of the scattering matrix of randomly oriented particulate matter (nanoparticles, aerosols, hydrosols etc.) can be measured in the laboratory by using laser light scattering experiments [1]. A number of different experimental studies have been carried out in the past to investigate the scattering behavior of particulate matter. Measurements of the elements of the scattering matrix of water droplets were performed by Pritchard and Elliot [2] in 1960 successfully. A sophisticated, fully computerized setup to investigate laser light scattering was built in the 1980 s by Stammes, Kuik and Volten, at the Department of Physics and Astronomy, Free University, Amsterdem [3]. The setup was subsequently improved and extended by Hovenier [4]. Some more advanced experimental 
setups are described by Barkey, Valentine et al. etc. [5,6]. However, the standard techniques used to characterize micron and sub-micron size particles [7] do not have the fine resolution needed for nano-sized particle detection because of which exploration of nanoparticles by light scattering tools has not yet been realized to their fullest potential. Here in this paper we are reporting for the first time, an attempt to characterize wide bandgap ZnS semiconductor nanoparticles by using a designed and fabricated diode laser based probing setup with indigenously developed data acquisition software.

\section{Experimental detail}

In this work, we begin with the study of light scattering properties of wide band gap ZnS semiconductor nanoparticles embedded in Polyvinyl Alcohol (PVA) matrix. Polyvinyl Alcohol (Aldrich) was selected as the host polymer matrix as it is an optically transparent aliphatic polymer having refractive index of 1.55 and dielectric constant of 2.0. It melts at $413 \mathrm{~K}$ and its density is around $1.08 \mathrm{~g} / \mathrm{ml}$. For the preparation of the matrix, a $2.5 \mathrm{wt} \%$ PVA solution was prepared in double distilled water [8], by stirring in a magnetic stirrer with stirring rate at $\sim 200 \mathrm{rpm}$ at a constant temperature of $70^{\circ} \mathrm{C}$ until a transparent solution is formed. Now, an aqueous solution of $0.15 \mathrm{M} \mathrm{ZnCl}_{2}$ was prepared. The aqueous solution was mixed into as prepared $2.5 \mathrm{wt} \%$ optically transparent PVA matrix in a ratio $1: 2$ and stirred for 6 hours at the same stirring rate as before but this time maintaining the temperature at $65^{\circ} \mathrm{C}$. This solution is treated with freshly prepared $\mathrm{Na}_{2} \mathrm{~S}$ solution and left in a cool and dark condition $[9,10]$. Colourless or faint milky coloured solution containing ZnS nanoparticles was obtained after 24 hours. As the experiment was done in differential mode, PVA samples with and without nanoparticles were prepared in specially prepared sample holders of size $1 \mathrm{sq} \mathrm{cm}$. XRD measurements, to get qualitative estimation of the nanoparticles were carried out with a Rigaku X-ray Diffractometer (Model : MINIFLEX). To get the surface morphology, along with the size, of the ZnS nanoparticles, a Digital Transmission Electron Microscope (Model : JEOL JSM 100CX) was used.

Next a laser based setup was designed to study the morphological characteristics of spherical ZnS nanoparticles. The schematic diagram of the experimental setup is shown in Figure 1. The setup consists of diode laser source (Melles Griot), controlled sample holders, photodetector arrangements, data acquisition systems and associated instrumentation. Light beam from a diode laser (Red, $\lambda=635 \mathrm{~nm}, 3 \mathrm{~mW}$ ) passes through a linear polarizer (Melles Groit, dia. $25 \mathrm{~mm}$ ). The polarizer placed in a rotating mount with a vernier dial is used to select either the perpendicular or parallel polarization component of the laser beam to use in the experiments. The laser light is scattered by a sample of nanoparticles embedded in the transparent PVA matrix which is suspended at the centre of the circular disc by a mechanical arrangement. The scattered light intensity is sensed by 16 highly sensitive static $\mathrm{Si}$ detectors 


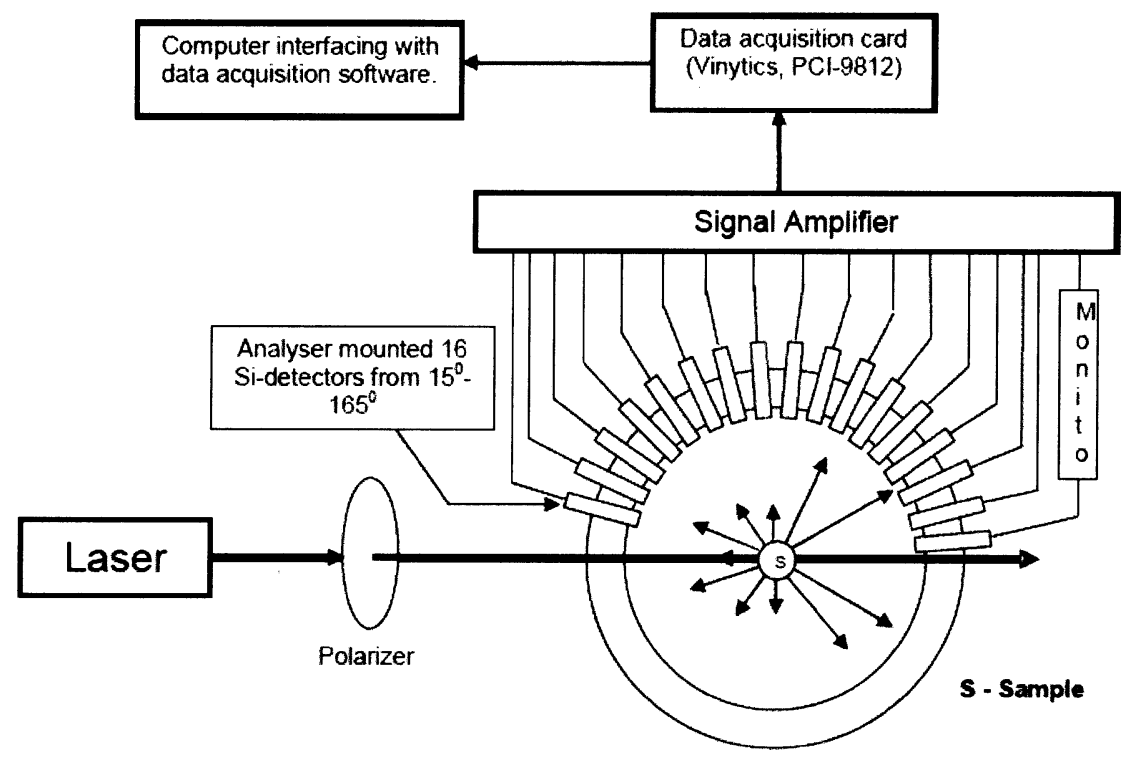

Figure 1. Schematic diagram of light scattering measurement setup.

(BPW34) mounted on a circular disc and which are connected to high gain, low noise OPAMP (LM 30IAN) based amplifier circuit. The amplified signals are interfaced to a dedicated data acquisition system (Vinytics, PCl-9812) for data recording. Each detector is separated from the next one by an angular distance of $10^{\circ}$. The whole array of 16 detectors can be rotated simultaneously about an axis perpendicular to the plane of the circular disc. Analyzers optimized for the diode laser wavelength is used to determine the state of polarization of the scattered light after it reaches the detector. The whole set up is covered by a black polished metallic enclosure to cutoff electromagnetic noise and beam stops are placed at strategic points to minimize the intensity of stray reflections. The experiments were carried out in differential mode [11], that is, scattered light intensity was initially measured from blank PVA matrices which did not have embedded nanoparticles and then measurements were made of the light intensity scattered from PVA matrices having embedded nanoparticles.

The data obtained is then plotted for analysis by comparing the data with theoretically generated Mie and T-matrix plots for particles of the same size till the experimental plot fairly follows the theoretical plots. The size of the particles are deduced from the agreement of the experimental and theoretical results only after taking the average of a large number of repetitions of the experimental observations to ensure accuracy of the investigations.

\section{Results and discussion}

X-Ray diffraction analysis for particle identification and microstructural characterization of nanoparticles has shown peaks at Bragg angles around $28^{\circ}$ and $45^{\circ}$ (Figure 3 ) 
confirming the presence of $\mathrm{ZnS}$ particles as it is known that $\mathrm{ZnS}$ show distinct peaks at $28.5^{\circ}, 47.5^{\circ}$ and $56.2^{\circ}$.

Transmission electron microscopy (TEM) technique was employed to observe the physical morphology of the embedded nanoparticles in the matrix. From the TEM pictures (Figure 4) the ZnS nanoparticles are found to be spherical and in cluster form. The average size of the individual nanoparticles was approximately $70 \mathrm{~nm}$.

From the graph (Figure 2) we see that the experimental plot is agreeing with the theoretical Mie plot within an acceptable limit of deviation. We also see that the experimental plot almost follows the $T$-matrix plot for $\mathrm{ZnS}$ particles of the same size as the Mie plot verifying the determined size of $70-80 \mathrm{~nm}$.

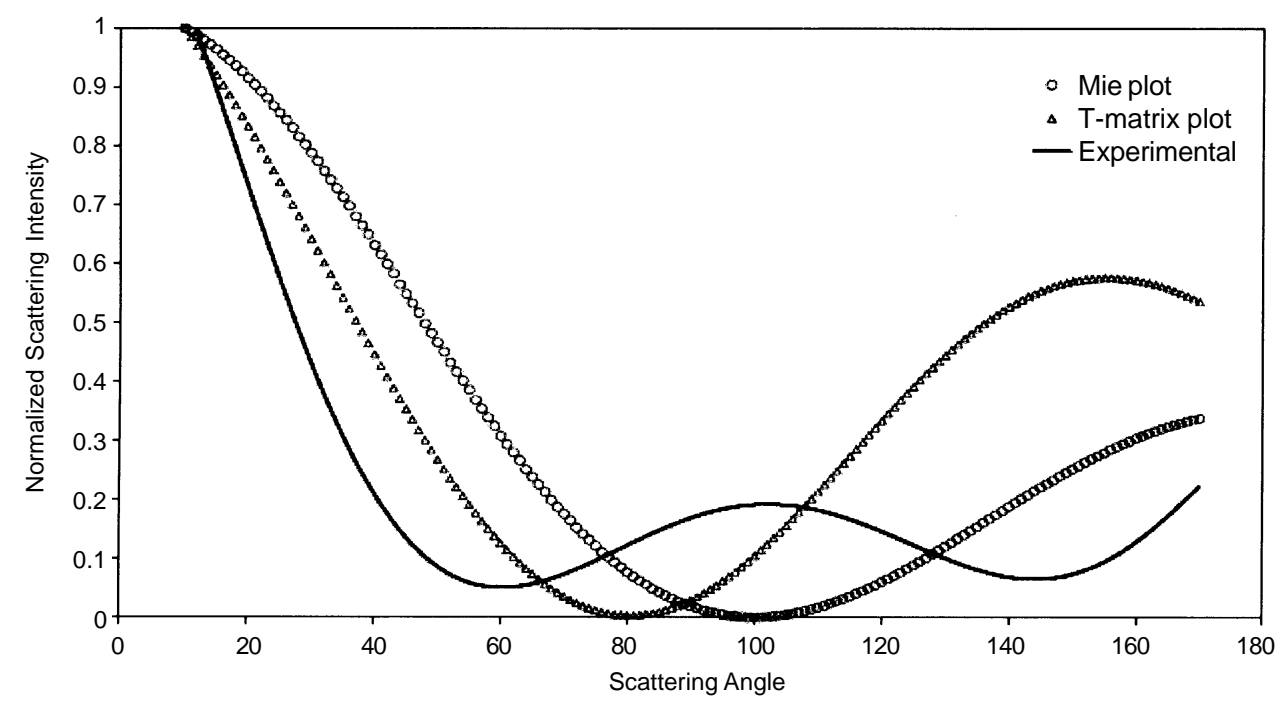

Figure 2. Experimental and theoretical graphs for particle size $70 \mathrm{~nm}$.

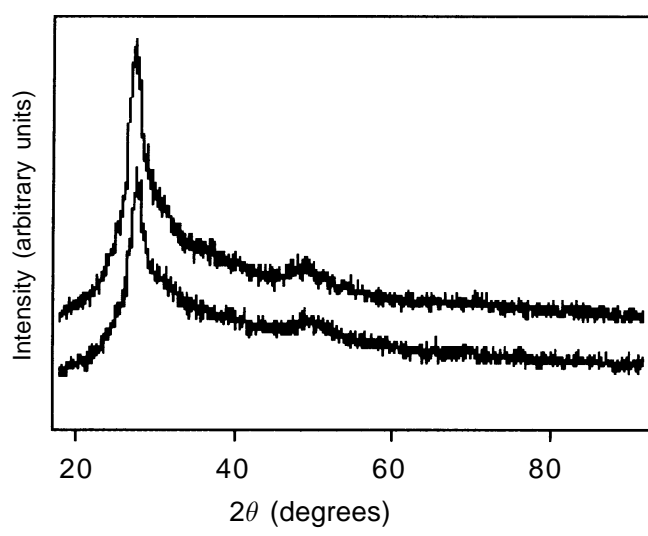

Figure 3. XRD pattern of PVA/ZnS nanoparticles system.

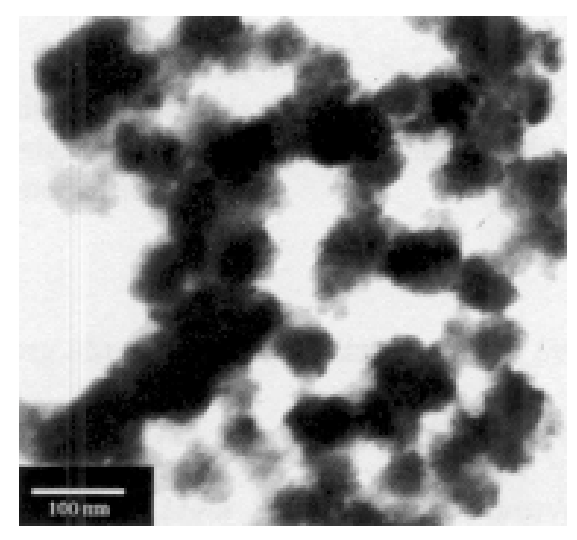

Figure 4. TEM image pattern of $P V A / Z n S$ nanoparticles system. 


\section{Conclusion}

The investigations have led to several important conclusions. The observations have proved the setup to be successful in design and fabrication for in situ measurement of light scattering characteristics of small particles from $10^{\circ}$ to $170^{\circ}$ which includes forward scattering and backscattering. Moreover the experimental results are consisteutly well matched with results drawn from established Mie theory and T-matrix approach. The minor deviation of the experimental plot from the theoretically generated plots in Figure 2 can be attributed to the superimposition effect of the dominant scattering pattern from individual spherical ZnS nanoparticles with the scattering pattern of a lesser intensity due the non-spherical cluster formation of the ZnS nanoparticles. Least square method gives a root mean square deviation of $\sigma_{M}=0.258892092$ for Mie plot and a root mean square deviation of $\sigma_{T}=0.41043136$ for $T$-matrix plot. Hence we can conclude that Mie theory is a better method for the analysis of our experiments. Again, the instrument reported here uses Diode laser and Si PIN photodiode which makes it lightweight, highly compact, and energy efficient. Also, preliminary results show that the scattering profile of the ZnS nanoparticles $(70-80 \mathrm{~nm}$ ) has a sharp forward scattering pattern followed by distinct scattering patterns around $100^{\circ}$ and $170^{\circ}$ (backscattering).

As the instrument has proved itself to be quite suitable for performing light scattering experiments on small particulate matter, extensive investigations will be further carried out on other metallic and semiconductor nanoparticles and aerosols.

\section{Acknowledgments}

We are grateful to Mr. Upamanyu Das, Dr. D Mohanta, Dr. K Baruah, Ms. S Sarmah and Ms. N Dutta, Department of Physics, Tezpur University for many fruitful discussions. We are also grateful to the technical staff of the Workshop, Department of Electronic Design and Technology, Tezpur University for the sincere help they provided during the making of the experimental setup.

\section{References}

[1] M I Mishchenko, L D Travis and A A Lacis Scattering Absorption and Emission of Light by Small Particles (Cambridge : Cambridge University Press) (2002)

[2] B S Pritchard and W G Elliott J. Optical Society of America 50 (3) 191 (1960)

[3] M I Mishchenko, J W Hovenier and L D Travis (eds.) Light Scattering by Nonspherical Particles : Theory, Measurements and Applications (San Diego Calif : Academic Press) (2000)

[4] J W Hovenier, H Volten, O Muñoz, W J Van der Zande and L B F M Waters J. Quantitative Spectroscopy and Radiative Transfer 79741 (2003)

[5] B Barkey and K N Liou J. Atmospheric Sciences 56605 (1999)

[6] M T Valentine, A K Popp and D A Weitz Optics Letters 26 (12) 15 (2001)

[7] Jinian Shu, Kevin R Wilson, Musahid Ahmed and Stephen R Leone J. Chem. Phys. 124030707 (2006)

[8] D Mohanta and A Choudhury Physica E27 176 (2005)

[9] D Mohanta, S S Nath, N C Mishra and A Choudhury Bull. Mater. Sci. 26289 (2003)

[10] S Chowdhury, D Mohanta, G A Ahmed, S K Dolui, D K Avasthi and A Choudhury J. Luminescence 114 95 (2005)

[11] J D Das, G A Ahmed, G K D Mazumdar and A Choudhury Asian Journal of Physics 10 (3) 323 (2001) 